\title{
Evaluación de riesgo en Caries de la Infancia Temprana
}

Rossana Sotomayor Ortellado

\section{Resumen}

El objetivo del presente estudio fue realizar una revisión sistemática de la literatura sobre la evaluación de riesgo en caries de Ia infancia temprana (CIT). Para ello fueron localizados por búsqueda electrónica treinta artículos científicos originales que cumplieron con los criterios de inclusión definidos. Los mismos fueron leídos de forma minuciosa para primero ser clasificados en aquellos que cumplen con aspectos básicos del método científico, y aquellos que no; luego, se identificaron en ellos indicadores y factores de riesgo puestos a prueba para verificar su asociación con la enfermedad en cuestión, a su vez, estos fueron clasificados sucesivamente en estadísticamente significativos o no; en aquellos con y sin coherencia en la literatura consultada $\mathrm{y}$ en provenientes de estudios longitudinales y transversales. También se determinó el número de estudios incluidos que contemplaron lesiones cavitadas y no cavitadas en la medición de la prevalencia o incidencia de la enfermedad.
Resultados: Un total de 103 factores e indicadores de riesgo fueron identificados, de los cuales, solo ocho provienen de los seis estudios correctos en cuanto a los aspectos básicos del método científico establecidos, y se mostraron acordes a los criterios utilizados para determinar su valor relativo como evidencia científica en el proceso de clasificación. $62 \%$ de los artículos incluidos contemplaron lesiones cavitadas y no cavitadas. Conclusión: Los indicadores y factores de riesgo que se ajustaron a los criterios establecidos en relación a los padres o encargados son: Historial de Caries; motivo de consulta con el Odontólogo: Necesidad de tratamiento; creencia fatalista sobre salud oral y actitud poco favorable con respecto a la prevención; En tanto que los relacionados a los niños son: Peso del niño/a elevado para la edad; Consumo elevado de bebida carbonatada; A mayor edad; Frecuentar guarderías públicas

Palabras claves: Caries,riesgo,revisión sistemática

\section{Artigo Original}

\section{Avaliação de Risco de Cárie na Primeira Infância}

\section{Resumo}

O objetivo deste estudo foi realizar uma revisão sistemática da literatura sobre avaliação de risco de cárie na primeira infância (CPI). Nesta busca eletrônica foram localizados trinta artigos científicos originais que atenderam aos critérios de inclusão definidos. Eles foram lidos de forma 
minunciosa para primeiro serem classificados naqueles que atendem a aspectos básicos do método científico, e aqueles que não; então, foram identificados entre eles indicadores e fatores de risco testados para verificar sua associação com a doença em questão, que por sua vez foram classificados sucessivamente em estatisticamente significativo ou não, e naqueles com e sem consistência na literatura. consultada, e em provenientes de estudo longitudinal e transversal. Também foi determinado o número de estudos que considerou lesões não cavitadas e cavitadas na medição da prevalência ou incidência da doença. Resultados: Um total de cento e tres fatores de risco e indicadores foram identificados, dos quais apenas oito dos seis ensaios estavam de acordo com os critérios e princípios básicos do método científico estabelecido, e estavam em linha com os critérios utilizados para determinar seu valor relativo, como prova no processo de evidência científica. $62 \%$ dos artigos considerou lesões cavitadas e não cavitadas. Conclusão: Os indicadores e fatores de risco que foram ajustados com os critérios estabelecidos em relação aos pais ou responsáveis são: história anterior de cárie, motivo da consulta com o dentista, necessidade de tratamento, conhecimento sobre saúde bucal e atitude desfavorável em relação a prevenção; Com relação as crianças foram relacionados: peso da criança elevado para a idade, consumo alto de refrigerantes e sucos; ser o mais velho, frequentar creches públicas.

Palabras claves: Cárie; risco; revisão sistemática.

\section{Original article}

\section{Caries risk assessment in early childhood}

\section{Abstract}

The aim of this study was to conduct a systematic review of the literature on risk assessment in Early Childhood Caries (CIT). This for, thirty original scientific articles that met inclusion criteria were localized by electronic search. They were read very thoroughly to first being classified into those that meet basic aspects of the scientific method, and those that do not; then they were identified risk indicators and risk factors tested to verify its association with the disease; these were classified in statistically significant or not, in those with and without consistency in the literature and in coming from longitudinal or transverse studies. It was also determined the number of included studies that looked for noncavitated and cavitated lesions in the measuring of the prevalence or incidence of the illness. Results: A total of 103 risk factors and risk indicators were identified, of which only eight were from the six studies that were adjusted to the basics aspects of the scientific method established, and were in line with the criteria of greater weight as scientific evidence in the classification process. $62 \%$ of the articles looked for cavitated and non-cavitated lesions. Conclusion: The risk indicators, and risk factors that were adjusted to the established criteria in relation to parents or guardians are: Caries record; reason for consultation with the dentist: The need for treatment; fatalistic oral health beliefs and unfavorable attitude about prevention; while related to the children are: Child's weight high for his/her age; High consumption of carbonated beverage; Increasing age; to assist to public kindergartens. 
Key words: caries, risk, systematic review.

\section{Introducción}

La caries dental en niños de 5 años o menos recibe diferentes denominaciones en la literatura ${ }^{1}$, es por ello que a modo de referencia, y por su carácter incluyente, la definición utilizada en el presente trabajo fue: Caries de la infancia temprana (CIT), considerada como la presencia de una o más piezas con lesiones de Caries (cavitadas o no cavitadas); piezas perdidas (a causa de la caries dental) o piezas restauradas, en dientes temporarios en niños de setenta y un meses o menos. ${ }^{2}$

En Latinoamérica la prevalencia de CIT es diversa de país a país, e incluso entre ciudades de un mismo país, ejemplo de ello son los trabajos de Cabral de Melo et al que en la ciudad de Recife, Brasil hallaron en una muestra de 3000 niños un $92 \%{ }^{3}$; mientras que Ribeiro et al en Joao Pesoa, en 224 niños un $43.7 \%$. En Medellín- Colombia, Saldarriaga verificó en 383 niños la elevada cifra de $74.9 \%{ }^{5}$. En tanto que en el Paraguay, en la encuesta nacional del Ministerio de Salud y Bienestar Social, con el apoyo de la OPS, se verificó en 691 niños $71.6 \%$ de caries sin tratar. $^{6}$

En prevención, la identificación de individuos o poblaciones en riesgo es ideal, para el mejor aprovechamiento de los recursos disponibles; Este enfoque resulta en ganancias tanto en términos de salud, como económicas. La evaluación incorrecta de riesgo puede hacer que se incurra en errores que tienen un costo tanto para el que provee el servicio, como para el que lo recibe; puesto que se pueden obviar medidas necesarias o por el contrario realizar más de lo requerido..$^{7-8}$

Para tener un panorama actualizado acerca de la evaluación de riesgo en la CIT, se recurrió a la lectura crítica de artículos originales, los mismos debían establecer asociación entre la prevalencia o incidencia de la enfermedad e indicadores o factores de riesgo. Para verificar la calidad de los artículos fue desarrollada una lista de siete ítems basados en criterios preconizados por Estrela, quien en su capítulo "Leitura Crítica de artigos científicos" propone un sistema para la identificación de publicaciones científicamente saludables y clínicamente válidas ${ }^{9}$, se resumió el análisis de los artículos definiendo dos ítems para evaluar el objetivo de los trabajos y cinco para evaluar el material y método empleados; de esta manera los estudios fueron clasificados según cumplían o no con los aspectos básicos del método científico establecidos. Debido a las diferentes definiciones operacionales encontradas para la lesión caries en las investigaciones, también fueron clasificadas según consideraban a las lesiones de la caries como cavitadas y no cavitadas o, solo cavitadas.

Ya en la obtención de datos de los artículos, se pudo observar que referentes clásicos para determinar el riesgo como la Lactancia, que por años ha sido responsable del nombre de la enfermedad "caries de Biberón o caries de la lactancia"1 no mostró poseer el peso en el eventual desarrollo de la enfermedad que aún muchos profesionales le atribuyen ${ }^{4 ; 10-12}$. En cuanto a: Edad de los niños, es indiscutible su valor de referencia, la literatura actual refuerza de forma contundente que a mayor edad, es mayor la prevalencia de la enfermedad; pero se hace hincapié en el hecho de que no resulta de gran utilidad para identificar individuos en riesgo en esta etapa, puesto que sería un contrasentido esperar que pasen los años y que la población sea pasible de enfermar, sin que se tomen medidas ${ }^{3 ; 5 ; 13-16}$. Otras variables como: consumo elevado de bebidas carbonatadas $^{16-20}$ y creencia fatalista sobre salud oral ${ }^{17 ; 18 ; 21}$ mostraron un buen desempeño para determinar 
la mayor posibilidad de padecer de CIT por los niños, entre el total de 103 variables identificadas en los trabajos incluidos.

La presente revisión de la literatura, podría ser una referencia, respaldada en evidencia científica actual, en la elaboración de estrategias para identificar individuos o población en riesgo; Como también podría constituirse en una base teórica para futuras investigaciones en el área.

\section{Objetivo}

Realizar una revisión sistemática de la literatura sobre la evaluación del riesgo en caries de la infancia temprana.

\section{Material y Métodos}

Fueron localizados 30 artículos originales a través de motores de búsqueda electrónica en bases de datos del área de la salud disponibles en la web (BVS, Scielo, Pubmed), los mismos cumplieron con los siguientes criterios: eran artículos científicos originales; poseían medición de la incidencia o prevalencia de la caries dental asociada a indicadores o factores de riesgo de la enfermedad; eran publicaciones de los años 2000 al 2010; estaban escritos en Español, Inglés o Portugués y por último, la edad de los niños incluidos en la muestra estaba comprendida entre los 0 y 5 años.

\section{Palabras claves utilizadas para la localización de artículos}

Mesh headings: Dental Caries; Risk factors; Child, preschool; Infant

Mesh qualifiers: Prevalence; Incidence
Otros términos en inglés no indexados: Early childhood Caries; Risk indicator

Descriptores DeCS: Caries Dental/ Cárie dentária; Preescolar/Pré-escolar

Calificadores DeCS: Prevalencia/ Prevalência; Incidencia/ Incidência

Otros términos en español no indexados: Caries de la infancia temprana; Indicador de riesgo; Factor de riesgo

Otros términos en portugués no indexados: Cárie Precoce na Infância; Indicador de risco; Fator de risco

\section{Criterios utilizados para clasificar a} los artículos según cumplían o no con aspectos básicos del método científico (Metodología elaborada en base a conceptos preconizados por Estrela 9)

Objetivo. 1) Claro: Específica en términos concretos que midió; No lo contamina con la metodología. 2) Preciso: Evita ambigüedades.

Material y Métodos. 1) Tamaño de la muestra: Menciona el origen para el cálculo del tamaño de la muestra. 2) Calibración inter e intra-examinadores: Menciona los procedimientos de calibración. Los valores de concordancia aceptados se ubican por encima del 85\%. (Valores Kappa entre 0,61 y 0,8) 3) Criterios de selección de la muestra: Describe claramente el método de selección de la muestra. 4) Instrumentos e Índices validados: Describe el proceso a través del cual validó el cuestionario utilizado para explorar los indicadores y factores de riesgo. Criterios utilizados para el diagnóstico de caries con referencia bibliográfica. 5) Definición de las variables: Detallada en la metodología. 
Identificación de Indicadores y Factores de riesgo y determinación de aquellos con mayor peso como evidencia científica: Fue elaborada una planilla tipo Excel y posteriormente se clasificó los datos de forma consecutiva en: Estadísticamente significativas o no; con y sin coherencia en la literatura consultada y en indicadores y factores de riesgo.

Definición de las variables utilizadas en la clasificación datos: Indicador de Riesgo: Variables encontradas en estudios de corte transverso; Factor de Riesgo: Variables encontradas en estudios longitudinales; Estadísticamente significativo (S): Poco probable que la asociación observada sea debida al azar; Estadísticamente no significativo (NS): Probable que la asociación observada sea debida al azar; Variables con coherencia (CC): Aquellas que fueron S o NS para el total de los estudios incluidos en la revisión; Variables sin coherencia (SC): Aquellas que fueron encontradas S y NS de manera simultánea en el total de los estudios incluidos en la revisión.

\section{Resultados}

Fueron identificadas un total de 103 variables para evaluar el riesgo de CIT en los 30 artículos incluidos. Tan solo seis estudios cumplieron con los aspectos del método científico establecidos, los mismos se encuentran resumidos en el cuadro 1, donde se puede apreciar a los autores, los indicadores y factores de riesgo (variables) en ellos encontrados estadísticamente significativos; la coherencia verificada para la variable identificada (CC) o falta de ella (SC).

En el gráfico 1 se puede apreciar que 18 estudios de los estudio incluidos en la revisión (62.0\%) consideraron lesiones cavitadas y no cavitadas para medir la variable Caries; en tanto que aquellos que consideraron como variable caries exclusivamente a las lesiones cavitadas fueron $11(37.9 \%)$. Nótese que uno de los 30 artículos no fue incluido en el cálculo, puesto que no se pudo esclarecer los criterios utilizados en él, a pesar de haber intentado localizar la referencia bibliográfica brindada.

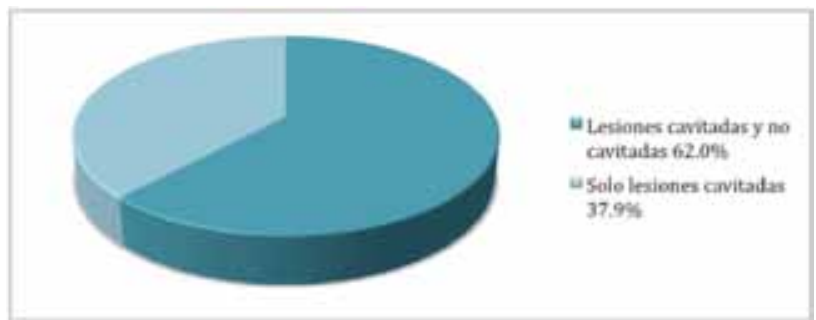

Gráfico 1. Criterios para el diagnóstico de lesiones de caries, utilizados en los estudios incluidos en la presente revisión. $n=29$.

\section{Discusión}

El cuidado de la salud dirigido a un grupo específico, referido como enfoque de riesgo se ha vuelto primordial debido a los altos costos que implica el mantenimiento de la salud, a lo que se le suma la restricción de recursos7.

Un mismo indicador o factor de riesgo es investigado de diversas maneras en cuanto a su asociación con la CIT, razón por la cual el número de variables identificadas en la presente revisión fue elevado; No obstante, es posible verificar que las mismas pueden ser agrupadas en temas ejes, tales como: Lactancia/Dieta, Higiene, Factores socio demográficos, Conductas y creencias con relación a la salud oral, Factores relacionados con S. mutans y el Uso de Flúor. ${ }^{3-5 ; 7-8 ; ~ 10-21 ~}$

Cabe destacar que la metodología de referencia otorga mayor peso como evidencia científica a estudios longitudinales frente a los de corte transverso ${ }^{9}$, por lo que la discusión se centrará en los artículos que correspondan a esta primera 
Cuadro 1. Factores e indicadores de riesgo encontrados estadísticamente significativos en los seis artículos con aspectos básicos del método científico correctos.

\begin{tabular}{|c|c|c|c|}
\hline Autor y año de publicación & Fuente & Factor/ Indicador de riesgo & $\begin{array}{l}\text { Coherencia } \\
\text { entre artículos }\end{array}$ \\
\hline \multirow{3}{*}{$\begin{array}{l}\text { Gonzalez- Martinez F et al. } \\
2009\end{array}$} & \multirow{3}{*}{$\begin{array}{l}\text { Rev Salud } \\
\text { Pública }\end{array}$} & Historial de caries de los niños. & $\mathrm{CC}$ \\
\hline & & El consumo diario de dulces & $\mathrm{SC}$ \\
\hline & & No exposición a fluorizaciones & $\mathrm{SC}$ \\
\hline \multirow{7}{*}{ Ismail A et al. 2009} & \multirow{7}{*}{ J Dent Res } & $\begin{array}{l}\text { Elevado consumo de bebidas carbonata- } \\
\text { das con contenido de azúcar }\end{array}$ & $\mathrm{CC}$ \\
\hline & & A mayor edad de los niños & $\mathrm{CC}$ \\
\hline & & Peso del niño/a elevado para la edad & $\mathrm{CC}$ \\
\hline & & $\begin{array}{l}\text { Motivo de consulta con el odontólogo (de } \\
\text { padres o encargados): tratamiento }\end{array}$ & $\mathrm{CC}$ \\
\hline & & $\begin{array}{l}\text { Historial de caries de los responsables y } \\
\text { los niños }\end{array}$ & $\mathrm{CC}$ \\
\hline & & $\begin{array}{l}\text { Creencia fatalista con respecto a la salud } \\
\text { oral }\end{array}$ & $\mathrm{CC}$ \\
\hline & & Ser niña & $\mathrm{SC}$ \\
\hline \multirow{5}{*}{ Cabral de Melo M et al. } & \multirow{5}{*}{$\begin{array}{l}\text { Cad. Saúde } \\
\text { Pública }\end{array}$} & A mayor edad de los niños & $\mathrm{CC}$ \\
\hline & & $\begin{array}{l}\text { Consumo diario de dulces entre las comi- } \\
\text { das }\end{array}$ & $\mathrm{SC}$ \\
\hline & & $\begin{array}{l}\text { Motivo de consulta con el odontólogo (de } \\
\text { padres o encargados): tratamiento }\end{array}$ & $\mathrm{CC}$ \\
\hline & & Baja escolaridad del encargado & SC \\
\hline & & Frecuentar guarderías públicas & $\mathrm{CC}$ \\
\hline \multirow{4}{*}{ Fadel C. 2009} & \multirow{4}{*}{ Tesis doctoral } & Número de hijos & $\mathrm{SC}$ \\
\hline & & Baja escolaridad de la madre & $\mathrm{SC}$ \\
\hline & & Bajo ingreso & $\mathrm{SC}$ \\
\hline & & $\begin{array}{l}\text { Alta frecuencia de visitas al cirujano den- } \\
\text { tista }\end{array}$ & $\mathrm{SC}$ \\
\hline Gomes I et al. 2006 & $\begin{array}{l}\text { Cad. Saúde } \\
\text { Pública }\end{array}$ & Baja escolaridad de los padres & $\mathrm{SC}$ \\
\hline \multirow{4}{*}{ Lin H et al. 2009} & \multirow{4}{*}{ Quintessence } & Presencia de biofilm & $\mathrm{SC}$ \\
\hline & & Frecuencia elevada de consumo de dulces & $\mathrm{SC}$ \\
\hline & & Bajo ingreso & $\mathrm{SC}$ \\
\hline & & $\begin{array}{l}\text { Actitud poco favorable con respecto a la } \\
\text { salud oral. }\end{array}$ & $\mathrm{CC}$ \\
\hline
\end{tabular}


clasificación y en aquellos que cumplieron con los aspectos básicos del método científico establecidos.

Con respecto a la lactancia, artificial o uso de mamadera Cabral de Melo et al; Zanata y Ribeiro et al no encontraron una asociación significativa con la Caries en infantes ${ }^{3-4,10}$; La segunda autora, a pesar de constatar que un $95 \%$ de las madres adicionaba a la leche, sacarosa $u$ otro similar a partir de los 6 meses. Cabe señalar, que sin llegar a tener significancia estadística, también constato que la frecuencia de ingestión de leche era mayor en los niños con Caries activas. En lo que respecta a Lactancia natural o del seno materno, Takuro et al verificó que si se extiende por periodos superiores a los 18 meses los niños son tres veces más proclives a tener Caries a los dos años de edad ${ }^{11}$; Thitasomakul et al también verificó como significativo el amamantamiento a los 18 meses $^{19}$; mientras que para Zanata no tuvo asociación significativa ni a los 12 , ni a los 24 meses $^{10}$. Van Palenstein et al por su parte, determinó que si el amamantamiento nocturno supera los 12 meses se convierte en un factor de riesgo; otro hallazgo en relación al tema, que para el mismo autor mostró influencia en la instalación de la enfermedad fue que el $98 \%$ de los niños de la muestra compartían la cama con la mamá ${ }^{12}$. El amamantamiento a libre demanda, reportada como práctica común en Tailandia y el sudeste de Asia podría ser un diferencial entre los niños con y $\sin$ caries $^{19}$. Iida reporta que las americanas de origen mexicano tienden a dar de mamar por periodos más largos que mujeres de otras etnias en los Estados Unidos de América ${ }^{13}$. Por lo expuesto, la literatura sugiere que no es la leche, aún con el agregado de azúcar, el factor de riesgo en sí, sino la manera en que se provee la misma, en relación a: frecuencia, tiempo total de lactancia, lugar donde duerme el infante.
En cuanto a otros aspectos de la dieta, a partir de la complementación alimentaria del infante, el consumo de bebidas carbonatadas fue $S$ para Fontana et al; Thitasomakul et al e Ismail et al. ${ }^{7,17-18} \mathrm{Y}$ para los estudios de corte transverso de Melendez et al y Montero et $\mathrm{al}^{16 ; 19}$, el consumo de hidratos de carbono fermentables, aunque de indudable relación causal, resulta difícil de ser valorado debido a la naturaleza subjetiva de los datos obtenidos a través de cuestionarios, mostrando alta falta de coherencia entre los estudios incluidos en la revisión ${ }^{3-5 ; 23-24}$. Tal fue el caso de Zanata, quien tuvo que prescindir de los datos obtenidos por el sistema de recordatorio de cuatro días debido a las inconsistencias en las repuestas de las participantes del estudio ${ }^{10}$.

Con respecto a la Higiene, la presencia de biofilm mostró un buen desempeño como determinante de riesgo aunque no mostró total coherencia entre los estudios, resultó $S$ para Zanata y Warren et $\mathrm{al}^{10 ; 15}$; NS para Fontana et al. $^{7}$ Estudios transversales que también encontraron significativa a esta variable (Pires do Santos et al ${ }^{14}$; Goldstein et $\mathrm{al}^{21}$; Fraiz et $\mathrm{al}^{22}$; Lin $\mathrm{H}$ et $\mathrm{al}^{23}$ y NS: Gonzalez- Martinez et al.) sin embargo, Frecuencia de cepillado fue hallado NS por: Zanata e Ismail et al y por los siguientes autores de estudios transversales: Pires do Santos et al ${ }^{14}$; Meléndez ${ }^{16}$; Tiano; Saldarriaga ${ }^{5}$; Gomez ${ }^{25}$; Mazhari.

El grado de instrucción de los progenitores es una referencia del componente social utilizado a menudo en estudios epidemiológicos. Aquellos estudios que intentaron determinar la asociación de le enfermedad en cuestión con el grado de instrucción de la madre no mostraron coherencia puesto que Thitasomakul et al y Zanata lo hallaron S; mientras que Ismail et al lo halló NS. En cuanto al grado de instrucción del padre, variable encontrada solo en los estudios de corte transverso incluidos, fue $S$ para Fraiz et $\mathrm{al}^{22}$ y para Gomes et $\mathrm{al}^{25}$ 
Ismail et al consideran que las preguntas acerca de creencias con respecto a la Salud oral podrían convertirse en prácticas herramientas para identificar a individuos en riesgo en atención primaria para la salud, puesto que se podría prescindir de infraestructura para efectuar examen clínico, al respecto, la frase utilizada en su estudio "La mayoría de los niños eventualmente desarrolla Caries dental" resultó $S^{17}$; en tanto que para Fontana et al.: "Dientes malos son heredados de los padres" fue igualmente significativa. ${ }^{7}$

La no exposición al Flúor con significancia estadística para uno solo de los estudios incluidos ${ }^{5}$, frente a la no significancia para tres de ellos ${ }^{4,26-27}$, supone una gran incoherencia frente a la marcada evidencia en la literatura de el rol protector de dicha sustancia, este resultado puede deberse a su escaso uso en la faja etaria estudiada ${ }^{27}$, o a errores en el diseño del estudio tales como: definición inapropiada de la variable.

La determinación de la presencia temprana de S. mutans, aunque muy útil por ser considerado hasta hoy día un factor etiológico primario, comprende una intervención costosa, se presenta una alternativa con la exploración de conductas relacionadas a la transmisión del microorganismo, encontrada S por Fontana et al. ${ }^{7}$

Por último, tres aspectos importantes en la interpretación y uso de los datos del presente trabajo: Es importante evaluar lo expuesto teniendo en cuenta que la significancia estadística sola, no es garantía o sinónimo de calidad ${ }^{9}$, es por ello, que se le han sumado datos como: la coherencia entre los diferentes autores y la verificación del factor después de transcurrir un intervalo de tiempo (resultados de estudios longitudinales). El segundo aspecto que no se debe perder de vista, es la interacción que se da entre los diferentes factores enumerados y en tercer lugar, la influencia de la cultura, que resulta en variaciones para un mismo factor de una población a otra.

\section{Conclusiónes}

Los factores de riesgo que se ajustaron a los criterios establecidos para esta revisión fueron: Con respecto a los padres o encargados: Experiencia de Caries; Motivo de consulta con el Odontólogo: tratamiento; Creencia fatalista sobre salud oral. Y los Indicadores de riesgo: Actitud poco favorable con respecto a la salud oral. En tanto que los factores de riesgo asociados a los niños: Peso del niño/a elevado para la edad; Consumo elevado de bebida carbonatada y los indicadores: Mayor edad; Frecuentar guarderías públicas. Con respecto a la determinación de la prevalencia o incidencia existe una tendencia a incluir lesiones cavitadas y no cavitadas en los criterios de diagnóstico clínico de la enfermedad.

\section{Agradecimiento}

A la Dra. Ninfa Jacquett por la orientación metodológica a lo largo del proceso de ejecución del estudio.

\section{Referencias}


2- American Academy of Pediatric Dentistry. Council on clinical affairs. Adopted 2003. Revised 2007, 2008 Disponible en http:/ / www.aapd.org/media/policies_guidelines/d_ecc.pdf

3- Cabral de Melo M, Viera de Souza W, Carvalho de Lima Ma. Y Braga C. Fatores associados a cárie dentária em préescolares do Recife, Pernambuco, Brasil. Cad Saúde Pública.Rio de Janeiro. Mar 2011. 27(3): 471-485 Disponible en: http: / / www.scielo.br/pdf/csp/v27n3/08.pdf

4- Ribeiro AG, Feitosa A, Rosenblatt A. Cárie precoce na infância: prevalência e fatores de risco em pré-escolares, aos 48 meses na cidade de João Pessoa, Paraíba, Brasil. Cad. Saúde Pública. 2005. Disponible en: http: / / www.scielosp.org/pdf/ $\operatorname{csp} / \mathrm{v} 21 \mathrm{n} 6 / 06 . \mathrm{pdf}$

5- Saldarriaga A, Arango C, Cossio M. Dental caries in the primary dentition of a Colombian population according to the ICDAS criteria. Braz. Oral Res. vol.24 no.2 São Paulo Apr./June 2010. Disponible en: http:/ / www.scielo.br/scielo. php?pid=S1806-83242010000200014\&script=sci_arttext

6- MINISTERIO DE SALUD PÚBLICA Y BIENESTAR SOCIAL (Paraguay) y ORGANIZACIÓN PANAMERICANA DE LA SALUD. Encuesta Nacional de Salud Oral 2008.

7- Fontana M, Jackson R, Eckert G, Swigonski N, Chin J, Ferreira A, Ando M, Stookie G, Downs S, Zero D. Identification of caries risk factor in toddlers. J Dent Res 2010. Disponible en: http:/ /jdr.sagepub.com/ content/90/2/209.full

8- Mattos MA, Melgar RA. Riesgo de Caries dental. Rev Estomatológica Herediana 2004; 14 (1-2): 101-106 Disponible en: http: / / www.upch.edu.pe / faest/ publica/ 2004/vol14-n1-2-art20.pdf

9- Estrela C. Metodología científica. Ensino e Pesquisa em Odontologia. Editoras Artes Médicas Ltda. 1ra. Edición 2001

10- Zanata R. Evaluación de un programa de salud para gestantes sobre la experiencia de Caries de sus hijos. Tesis doctoral 2001. Disponible en: www.teses. usp.br/teses / disponiveis / 25/25131/tde.../RegiaZanata.pdf

11- Takuro Yonezu, Nagako Ushida, Masashi Yakushiji. Longitudinal Study of Prolonged Breast- or Bottle-fedding on Dental Caries in Japanese Children. Bull Tokio Dent. 2006. Disponible en: http://www.biomedexperts.com/ Abstract. bme/17510545/Longitudinal_study_of_prolonged_breast-_or_bottle-feeding_on_dental_caries_in_Japanese_children

12- Van Palenstein w, Soe W, van't Hof M. Risk Factors of Early Childhood Caries in a Southeast Asian population. J Dent Res 85(1):85-88, 2006 Disponible en: http:/ /jdr.sagepub.com/content/85/1/85.full.pdf

13- Iida H, Auinger P, Billings R y Weitzman. Association Between Infant Breastfeeding and Early Childhood Caries in the United States. Pediatrics, 2007. Disponible en: http:/ / pediatrics.aappublications. org/content/ 120/4/e944 .full

14- Pires dos Santos A, Mendes V. Caries prevalence and risk factors among children aged 0 to 36 months. Pesquisa Odontológica Brasileira. 2002. Disponible en: http:/ / www.scielo.br/scielo.php?pid=S1517-74912002000300004\&script=sci_arttext

15- Warren J, Weber-Gasparoni K, Marshall T, Drake D, Dehkordi-Vakil F, Dawson D, and Tharp K. A Longitudinal Study of Dental Caries Risk among Very Young Low SES Children. Community Dent Oral Epidemiol. 2009. Disponible en: http: / / www.ncbi.nlm.nih.gov/ pubmed/19046332

16- Menléndez Gustavo T. Estudio Epidemiológico de la prevalencia de caries y su relación con los hábitos alimentarios y de higiene bucal en los niños de 6 a 36 meses de edad. Disponible en: http:/ / www.ceo.com.pe/005_revista_art01htm

17- Ismail A, Sohn W, Lim S and Willem J. M. Predictors of Dental Caries Progression in Primary Teeth. J Dent Res 88(3):270275, 2009.Disponible en: http: / /jdr.sagepub.com/content/88/3/270.abstract

18- Thitasomakul S, Piwat S, Theamontree A, Chankanka O, Pithpornchaiyakul W and Madyusoh S. Risk for Early Childhood Caries Analyzed by Negative Binomial Model. J Dent Res 2009; 88; 137. Disponible en: http://jdr.sagepub.com/ content/88/2/137.full

19- Montero D, López P, Castrejón R. Prevalencia de caries de la infancia temprana y nivel socioeconómico familiar. Revista Odontológica Mexicana. 2011. Disponible en: http: / / www.medigraphic.com/pdfs / odon/uo-2011/uo112d.pdf

20- Fadel C.B. Aspectos sócio-dentários relacionados à transmissibilidade materna da cárie dentária e a percepção das mães sobre suas causas e seu controle. Tesis doctoral. Universidad estadual Paulista. 2009. Disponible en: http://www. athena.biblioteca.unesp.br/exlibris/bd/bfo/33004021074P1/2009/ fadel_cb_dr_araca.pdf

21- Goldstein R, Gudiño S. Riesgos Nutricionales e Higiénicos Asociados a la Caries de la Temprana Infancia. Revista Científica Odontológica, Vol 3, No 2 (2007). Disponible en: http: / / colegiodentistas.org/ revista/index. php/revista odontologica / article/viewArticle/48/101 
22- Fraiz F.C,Walter L. Study of the factors associated with dental caries in children who receive early dental care. Pesqui. Odontol. Bras. vol.15 no.3 São Paulo July/Sept. 2001. Disponible en: http://www.scielo.br/pdf/pob/ v15n3/a05v15n3. pdf

23- Lin H, Zhang R, Lo E, Schwarz E. A case-control study comparing 30\% of children with the highest dmfs score and children with no caries in southern china. The Chinese Journal of Dental Research. 2009. Disponible en: http:/ / www.quintpub. com/userhome/cjdr/cjdr_12_1_\%20Lin_4.pdf

24- Gonzalez- Martinez F, Sanchez R, Carmona L. Indicadores de Riesgo para la Caries Dental en niños pré escolares de La Boquilla Cartagena. Rev salud pública. 2009. Disponible en: http:/ / www.scielosp.org/pdf/rsap/v 11n4/v11n4a13.pdf

25- Gomes I, Moreira R, Mazza M, Saliva S. Early Childhood Caries: The influences of sócio- behavioral varibles and health lócus of control in a group of children from Araraquara, São Pablo, Brazil. Cad. Saúde Pública. 2006. Disponible en: http:// www.scielosp.org/ scielo.php?pid=S0102-311X2006000600014script=sci arttext

26- Slabsinskiene E, et al Severe early childhood caries and behavioral risk factors among 3-year-old children in Lithuania. Medicina (Kaunas) 2010; 46(2) Disponible en: http:/ / www.ncbi.nlm.nih.gov/pubmed/ 20440088

27- Quiñones Ybarría María Elena, Lisbeth Pérez Pérez, Ferro Benítez Pedro Pablo, Martínez Canalejo Humberto, Santana Porbén Sergio. Estado de salud bucal: su relación con el estado nutricional en niños de 2 a 5 años. Rev Cubana Estomatol [revista en la Internet]. 2008 Jun [citado 2011 Mayo 30] ; 45(2): . Disponible en: http://scielo.sld.cu/scielo. php?script=sci_arttext\&pid=S0034-75072008000200004\& lng $=$ es

Recibido: 21-09-2011

Aceptado: 18-10-2011

Correspondencia: rossoortellado@yahoo.com 